\title{
UX \& FOMO. LOOKING FOR LOVE OR LOOKING FOR OPTIONS?
}

\author{
Mihail Vuzharov \\ New Bulgarian University \\ vuzharov@gmail.com
}

\begin{abstract}
:
This paper takes a look at the evolution of the Web and the digital products and services that were forged within it. It attempts to trace the progress of the internet's design, to outline its current status, and to forecast its potential development. It also discusses the ability of design to influence culture to an extent that exceeds its explicit and implicit objectives.
\end{abstract}

Keywords: FOMO, UX design, culture, digital services, instant gratification

Digital Age in Semiotics \& Communication, Vol. II, 2019, Pp. 78-92 https://doi.org/10.33919/dasc.19.2.5 


\section{The Web}

Remember when the Internet was hailed as a new force of freedom and democracy? How it was the Internet's destiny to turn everything around; to turn the world on its head; to redefine everything? Well, it did, in a way.

Information was to always be at our fingertips - unlimited and unmediated, ever fuller and ever more complete. It would flow freely, unchanneled, unfiltered, unbiased, uncorrupted, pure and chaste. It would be accessible to everyone - as long as they had internet access, but that has proven to be just a matter of time. And, most of all, freedom of expression was to be absolute.

The Web 1.0 - the first iteration of the Internet - was born in the 80s and 90s. It was a limited, albeit growing, network of computers, where increasing amounts of data were readily available but mostly un-organized. It was an infodump, where any service design was mostly task-centered and user interface (UI) was only starting to be of importance - the learning curve was still high; users had to depend on their own "encyclopedic competence" (Eco 1986) in order to navigate the chaos of information.

By the end of the 1990s, the Internet had matured - it was now Web 2.0 (DiNucci 1999), the "participatory web" (Blank and Reisdorf 2012) or the "social web" (Rheingold and Howard 2000). User-generated content had become a key digital commodity, populating the many new and constantly growing social media websites with free content, while letting them provide their services "for free" (users "paid" by being subjected to adverts) to the users. The information had morphed from an infodump into a library (or a dictionary) - a Porphyrian tree (Eco 1986). It was now structured and more readily accessible, demonstrating a more refined reliance on process-centered design and requiring perusers' "navigational competence" (Bankov 2010). Usability was becoming an important aspect of online services, as users had to feel at ease with any new service they were subscribing to, since a steep learning curve meant a higher bounce rate.

Fast-forward to today (2019) - the Web has evolved past its 2.0 designation: data is now presented in a personalized manner, respective to the particular user's personal encyclopedia; user experience (UX) design - a user-centric paradigm - has become a conscious and sought-after standard. Cloud-based services have become the de facto standard, with cloudbased operating systems not far behind. Power has been transferred to the companies providing the tools for the Web - a process that had begun with the growth of Web 2.0; whoever had the technology, had the control. Slowly, a few major players - the Digital Giants - became the de facto operating system of the Internet. Whether it is correct to call today's digital environment "Web 3.0" will become clear in the years to come - "Web 1.0", after all, 
was a retronym for the original "World Wide Web". While some do apply this consecutive moniker, others await a more thorough shift, a revision of ideals, to designate as Web 3.0.

Whether a fundamental shift - such as radical service decentralization and a return of personal data ownership to users - will be the future of the digital world is hard to say. However, the technological roadmap of the digital future appears to depend on a profound change - a shift of agency from human to machine: Artificial Intelligence (AI), Machine Learning (ML) and Deep Learning have become some of the most important fields in digital science and technology. Furthermore, the Internet of Things (IoT) and the succeeding Internet of Everything (IoE) are paradigms of digital interconnectedness, which have been transforming the promises of science fiction into daily occurrence. Essentially, the next Web will be a network - both abstract and physical - with its own mind.

\section{The Digital Giants (FAMGA)}

The Digital Giants (FAMGA) hold more than $40 \%$ of the US stock market. The acronym FAMGA stands for Facebook, Apple, Microsoft, Google and Amazon - the five biggest stocks in Nasdaq. They are major players in the shaping of the digital environment, current and future. This is, of course, a natural turn of events - the holders of the largest infrastructures and services, and of the most capital, cannot ignore the trends. In fact, they would always strive to shape trends and be among the avant-garde.

Since their inception, these companies have been shaping online behavior by creating and enforcing policies - most obviously via their End-user License Agreements (EULAs) and community standards, but also through UX design. These services are not just neutral media, as many tend to perceive them, but are rather "legislators" of digital life; they are digital institutions, policing and politicking users (Milan, 2015). They dictate what is appropriate behavior (no images of female nipples on Facebook or Instagram), but they also shape what actions are at all possible (remember when Facebook had just a "Like" button?). Digital life is ruled via code - as Lawrence Lessig put it, "code is law". What exists as technical abilities, technical code, UX design, affordances, algorithms, etc., dictates possible behaviors.

\section{Black Foam}

The rules that govern digital life are not always visible. In fact, it is only the EULAs and community standards that can be easily accessed and reviewed. UX design is much less visible, as only the user interface (UI) is a clearly discernible part of it - and even in this case it is hard to recognize 
the limitations it imposes on users. However, other aspects of UX - such as visual design choices, workflows, data organization, algorithms, goals, etc. - shape not only the experience, but also users' expectations. This blackbox-styled system, lacking discernible boundaries, is what Bernhard Rieder has dubbed "Black Foam" (Rieder 2005). The users see and experience the surface, but do not understand the depth and the machinery behind the UI - they don't know what happens in the background, nor why.

A much used example of this property of digital services is Google's PageRank - the ranking algorithm the search provider uses to rank pages it links to in the search results. Neither the algorithm itself, nor the parameters used are accessible, although Google is the largest search provider in the world. The reasons any page is ranked as it is in a Google search are opaque - off-limits. Additionally, the algorithms are frequently updated ${ }^{1}$ for two main reasons: to improve the search results, so that they get ever closer to the perfect answer for the question asked; and to ward off misuse of the algorithm (e.g. by Search Engine Optimization (SEO) specialists, who keep a close eye on Google and work hard to reverse-engineer its parameters in order to artificially improve the ranking of their entrusted websites).

\section{Personalization and the nature of algorithms}

Large players in the digital field yield more power than smaller players. A good example is the so-called Search Engine Manipulation Effect (SEME). This is where SEO lives - as well as publicity. Search results can be manipulated - even if not deliberately - as per popular sentiment or market forces:

Generally speaking, link analysis turns the power-law link structure of the Web, where a small number of hubs dominate a large number of scarcely linked sites into a measure of importance. The underlying principle has been called "cumulative advantage", "preferential attachment" or "Matthew effect", but the consequence is simply that already well-ranked sites have a higher visibility and therefore get linked more often, leading to yet better rankings. In other words, the rich get richer. Using popularity as measure for quality is, of course, a normative decision. The logic of the hit, combined with the fact that search engine optimization (SEO), link campaigning, and classic marketing allow economically potent actors to skew the game in their favor is effectively responsible for both centralization and commercialization tendencies. (Rieder 2009)

\footnotetext{
${ }^{1}$ https://moz.com/google-algorithm-change (accessed 2/4/2019).
} 
One improvement that Google has applied to its search service has managed to reduce this exploitation (or, at least, to impede it): the personalization of a search - essentially, providing search results that are relevant to the specific user, rather than providing results that are high-ranking in a general catalog. Coincidentally, this turn to personalization has also earned more points for Google, as providing more relevant results for the users has made it the most used search engine in the world. Of course, this has allowed the company to better monetize its services, as the company has applied the technology behind search personalization to provide ad personalization and improve targeting.

Personalization has become one of the most important features of our digital environment: from the user's point of view, it provides precisely the information or experience that the user requires, needs or expects; from the point of view of the media (or service) it provides an unprecedented efficiency in content triage, allowing for different adverts to appear in the same content, depending on the consumer; from the advertiser's point of view, it provides an unparalleled precision in consumer targeting, effectively shifting paradigms from the target group to the target user. And all of this is based on one main concept: the User Model.

As has already been discussed (Vuzharov 2018), while personalization has indeed managed to improve the end-users' experience, it has also defined a certain structure, a pre-set mode of information retrieval and communication: services such as those owned by FAMGA have achieved their current level of personalization by creating a complex model of the user and serving data (be it status updates and links, search query results, adverts, book suggestions, etc.) based on that model. The way the User Model is created generally relies on three (and judging by patents filed by some of the companies, soon to be four) main paradigms: user behavior, collaborative filtering, inference/extrapolation (and soon, disposition/emotion analysis).

Behavior analytics is a historical, statistical analysis based on past actions and preferences by the specific user. It requires the capture and aggregation of large quantities of raw data (signals) across all of the user's connected applications and devices. While a logged-in user is easier to track across multiple devices, it is still possible to collect plenty of information even about users who are not logged into the platform. Marketing and analytics services, such as Acxiom, have devised methods that allow them to match figures from different user sessions to aggregate immense volumes of data (Pariser 2011).

Collaborative filtering, on the other hand, relies on grouping users into clusters based on their similarities. It is based on overt social characteristics and processes, such as rating certain songs (e.g. on Spotify) or pur- 
chasing certain products (e.g. on Amazon). These user signals can then be interpreted as an approximation of the user's perception of the respective realm and her association (by similarity) to an abstract cluster of users who exhibit similar attitudes. Such filtering mechanisms largely power recommendation services, such as Spotify's Discover Weekly playlists; they look at a cluster of similar users and recommend highly rated items to those users who have not yet encountered them (or, at least, for whom there is no data regarding such encounters in their user profiles).

In contrast to collaborative filtering, inferential methods are based on covert social characteristics and processes, and often rely on psychographics and statistical data drawn from large populations. They are based on the analysis of hundreds of thousands of records in order to extrapolate additional details about users. An example might be the use of explicit Facebook page likes in order to reach certain conclusions (e.g. if the user likes Nutella, she is classified as a sweet-tooth, chocolate lover). However, this method also relies on implicit traits in order to classify users (e.g. if the user is male and his Facebook page-likes include "Wicked the Musical", the "No $\mathrm{H} 8$ " campaign, and other human rights campaigns, he may be classified as gay) (Kosinski, Stillwell and Graepel 2013).

Finally, we should mention the newest trend in user profiling, namely dispositional analysis. While this technology is still being tested and finetuned, it does show great promise (for marketing purposes). What this method aims to achieve is to recognize the user's current mood, in order to surface adequate information. It is yet another layer of the user model, which would allow an app or service to use the built-in webcam (or other available instrument) of a user's device, analyze her face (or why not typing speed, pulse, bodily temperature, etc.), and eventually display information that would harmonize with the user's putative emotional state (or would, in more unethical circumstances, take unfair advantage of her momentary emotional state).

Essentially, the entire premise of personalization is an approach built upon the user's observed behavior, her user model constantly being updated with regard to the persistent flow of new behavioral data. In other words, the user model is an attempt at a digitally reconstructed approximation of the user's individual encyclopedic competence and system of expectations. Respectively, personalization algorithms build upon these approximate reconstructions (Vuzharov 2018).

However, there is more to the nature of algorithms than just a User Model. In order for a model to be useful it must contain immense quantities of data, which need to be ordered for it to make sense. Certain properties are weighted more than others, attributes are hierarchized, traits are arranged 
in complex networks. This is a common approach to user modeling. However, when an algorithm works in the background, it is impossible for the user to identify what traits are important for a specific service, how they are ordered, what weights are applied to which qualities, etc. To put it very simply, search results for "formula" would be different if the User Model instance's quality "mother" was weighed higher than the quality "chemist".

Frequently, internet users do not realize that their search results are automatically "curated" by the service, based on their User Model. In fact, they don't seem to understand that each internet user experiences, in fact, her own version of the internet - from search results, to Facebook Newsfeed, to the prices of certain items in online shops (i.e. personalized pricing). While services like Netflix, Amazon and Spotify are actually expected and encouraged to provide highly personalized suggestions, users hardly ever pay due attention to areas of the internet where personalization doesn't feel to be all that important. They still seem not to recognize that search results are not natural - just as algorithms are not natural. They are man-made tools, which exist to serve a purpose (Vuzharov 2018).

\section{Perception of Information}

Essentially, our entire digital experience is based on a User Model, derived by various algorithms, deep learning mechanisms and AI systems - a veritable black foam, which, in turn, weaves a personalized and unique Dynamic Text for a very special Echian "model reader" - the "model user". Our personal Internet's Dynamic Text, then, would be authored by way of the User Model and intended to be experienced solely by the ideal actuation of the User Model, the Model User.

Naturally, one could argue that the better the algorithm, the better the model, and the more perfect the representation of the user. Essentially, the most perfect algorithm would be able to provide information which corresponds ideally to the user's own encyclopedic competence - no more, no less. Effectively, this is a self-perpetuating and self-limiting system. Such a structure would minimize serendipity, or chance discovery and productive error. The farther we depart from encyclopedic competence, and then from navigational competence (where we were at least able to browse into areas unknown), the farther we are moving from the unknown. Only information from the known universe will be available to the user, while all else sits, unattainable, behind an event horizon which, counterintuitively and almost paradoxically, shrinks around the user as the algorithms become ever better at knowing her.

Digital services users perceive their experience as disintermediated. Seldom do they even think about why and how certain information reaches 
them when they use digital services. As stated above, Google search results and Facebook Newsfeed items are personalized every time, for every user. These bits of information are more suggestion than discovery - or, rather, they could be qualified as guided discovery - as they are based on our respective User Model (Vuzharov 2018).

\section{Smartphones and UX}

Guided discovery and the perception or disintermediation have become an ever-present companion to the typical digital citizen. With the rise of smartphone ownership (a projected 3bn smartphones worldwide by $2020^{2}$ ), easy-to-use personalized services have become the norm - from local weather information, through dining suggestions, to traffic route recommendation. It is hard to believe that the modern smartphone is only eleven years old (as of 2018).

More powerful new processors, new and better screens, improved memory, enhanced batteries, faster charging - technological progress renders otherwise adequate older (aging) hardware much cheaper. Simultaneously, we tend to desire the newest, biggest, flashiest gadgets - we desire the novelty, the status, the gimmick. ("We are so eager to embrace the new digital technologies," as Prof. Dario Martinelli exclaimed in a presentation during New Bulgarian University's XXIII Early Fall School of Semiotics in Sozopol.) This has effectively made smartphones more accessible than ever - to such an extent that some regions have leaped over the PC and laptop age altogether, landing directly in mobile era. With a projected worldwide mobile internet penetration surpassing $64 \%$ by $2019^{3}$, digital services seem to be headed for world domination.

The spread of digital services to billions of users has scaled community to the level of society, much like the effect Benedict Anderson (2016) suggests capitalist printing exerted on the formation of nation states. Groups of people with similar interests now readily join one another, thanks to the affordances of contemporary digital communications. Suddenly, needs that were uncommon, and seldom catered to, have become noteworthy. New needs emerged as well, crystallizing amid unforeseen situations - by accident, as is often the case - or even born from new market paradigms. New technologies and approaches appeared to fill the vacuum and provide for those novel market needs. This development, paired with the intrinsic competitiveness of the open market, led to better services, better customer experience, and better user experience design.

\footnotetext{
${ }^{2}$ Statista.com

${ }^{3}$ Statista.com
} 
Usually, UX design is perceived as the field in service development dedicated to improving users' experiences - a paradigm in which UX design pledges allegiance to the user. "User experience design is the process of enhancing user satisfaction with a product by improving the usability, accessibility, and pleasure provided in the interaction with the product. User experience design encompasses traditional human-computer interaction (HCI) design, and extends it by addressing all aspects of a product or service as perceived by users." ${ }^{\text {"T }}$ The very development of UX design can be observed as one surveys the history of digital services, tracing the stages of the internet back to Web 1.0 - from task-centered design, through an increased reliance on usability, all the way to the current paradigm of user-centric thinking. In fact, design can first be located when humankind first started shaping rocks for use as rudimentary tools. User experience design is nothing but a continuation of that desire to make the world easier and more pleasant to use.

However, there is a contradiction within (most) UX design, one hidden in plain sight. It arises from the simple fact that most popular services in wide use (at least today) are created by for-profit organizations, whose main goal is monetization. The only major not-for-profit service currently in existence is Wikipedia, owned by the Wikimedia Foundation. The foundation relies mostly on grants and public contributions in order to keep its service running. This contrasts with other publicly available "free" services - such as Google Search, Google Documents, YouTube, Facebook, Instagram, WhatsApp, Twitter, etc. - in that the latter services have a primary, often publicly unstated (albeit clear) purpose - to generate revenue. And while UX design does have the satisfaction and comfort of the user as its primary goal, it in fact, has an intrinsic, a priori allegiance to the main objective of the organization that it serves - and that, to reiterate, is revenue generation. Thus, UX design can be described as a double agent, per its allegiance, but the practical mission of the parent organization will always trump the stated ideals that otherwise define UX design as a discipline.

Why, however, would design depend on the objective of some overarching commercial organization? The truth is, design is far from neutral - as Benedict Anderson theorizes, it has the power to define outcomes. As Anton Varlamov of the Institute of Higher Nervous Activity and Neurophysiology at the Russian Academy of Sciences commented at the XIII EFSS, "We train our skills by repetitively concentrating on certain tasks." Respectively, it can be argued that digital affordances can shape culture the way a system is designed will not only shape the way the system is used

\footnotetext{
${ }^{4}$ Wikipedia.
} 
but may also affect the broader culture that exists outside of that system. (Just imagine giving chopsticks to a child who has only ever seen spoons.)

As has been discussed elsewhere (Vuzharov 2018), these services do shape the rules for their users. A simple illustration would be Facebook's "Like" button - one of the UX affordances that Facebook's algorithms rely on, when modeling a user's NewsFeed. This button is utilized when a user wants to interact positively with a certain piece of information on Facebook, be it a status update, photo of a cat, a shared link, etc. This creates an instant semiotic contradiction - "things she likes" vs. "things she doesn't like". However, even with the recent introduction of Facebook "reactions" (the new ones being "Love", "Haha", "Wow", "Sad", "Angry") one will be unable to complete the semiotic square with the obviously missing "things she dislikes" and "things she doesn't dislike". Indeed, it would be hard to have the latter without the former, but "dislikes" are impossible to monetize in the existing ad-based profit model. Essentially, what we have here is an incomplete structure serving as the User Model's foundation, which is then used as the filter through which the user perceives the universe.

What we have depicted above is, essentially, a form of control. However, it must be stated that UX design is generally not (ab)used maliciously; rather, what we usually tend to observe is a healthy (from an economical point of view) pursuit of optimization and market domination, which, however, cannot be realized without the shaping of information delivery mechanisms. On the one hand, design must increase the (perceived) value for users, in order to attract them to and retain them within the service; and on the other hand, it must concentrate on ways to satisfy bidders for the advertising space offered on the services' real estate (note that earlier we used quotation marks when we talked about "free" services - this is because, in the general case, their users do indeed pay for access, however, instead of actual money, they surrender their personal information). Additionally, most services prioritize items with which the user seems more likely to interact, based on her User Model (Vuzharov 2018).

Design can actually shape our perception of happiness. But what is happiness in the digital realm? In our "sharing" (or, rather, "broadcasting") culture, happiness appears to be colorful, smiling, unblemished, retouched, photoshopped, smooth, bright... UX design acknowledges and encourages that - apps provide built-in filters, instant beautification algorithms in camera software, easy-to-use effects in Instagram, even special apps for enhancing physical characteristics on visual media (the author of this paper gets constant ads for apps that allow the user to install a six-pack on any shirtless picture they may have in their phone's picture album). This perception of happiness does not simply stay "online" - it seeps into our culture. 


\section{Device \& Service Branching}

The Internet used to be this place we entered. We would "go online". First, we would sit at a desktop and have a modem dial some number, as we entered the Web 1.0. Then, we (finally) got broadband, with better speed and constant connectivity - as long as we stayed where the router was - and it was much easier to go online. But today even services that used to be perceived as intrinsically offline (e.g. MS Office, Chrome OS) have become online services.

Today we go offline. The Internet is no longer a place we go - today it's things we do; a reality we live in. It's in our hands and our pockets, in our TVs, our fridges and our cars; it tells us the weather, it helps us get a cab, reminds us where we parked, prepares our shopping lists. We can just say "Siri, find me a recipe for brownies" or "Alexa, order 'Kant and the Platypus'?" Pretty soon digital services will be driving us around - physically - and picking the best route for us, with no need for a human in the driver's seat. (In fact, there has been speculation that the autonomous, electric cars of the near future will provide a "free" service - just the way that most digital giants today offer their services in exchange for our attention and personal information. Imagine a car taking you to your destination of choice but in exchange for the free ride, you would have to go via a personalized route, which will take you through certain shops, and may even take a mandatory break.) As Prof. Kristian Daneback from the University of Gothenburg exclaimed at the XIII EFSS, "Our society is becoming more and more digitally-centered".

As the Web developed from 1.0 to today's ubiquitous digital meta-reality, apps and connected devices experienced a similar trend. The shift to IoE goes hand in hand with a transition from AiOs (all-in-one devices) to a myriad of dedicated services and devices. We have witnessed the shift from the good-for-everything smartphone to a collection of specialized gadgets sports trackers, action cameras, personal medical devices, etc. It was software that led this shift, as object-specific mobile apps appeared first - for example, geolocation apps gave rise to jogging tracking apps, while social networks specialized, giving birth to phenomena like the dating app. Some of the most prominent dating services that appeared include Zoosk, Badoo, OkCupid, POF, Grouper, Tinder, Grindr, 3nder, Blendr, Scruff, etc... (A friend of mine jokingly exclaimed, "Such a vast infrastructure just for sharing nude photos!")

\section{FOMO}

All of these new apps demand our attention - it's an important characteristic of their design. Since they are all "free", they need to rely on active users, and to such an extent that one of the most important metrics for a certain 
digital service is the MAU - Monthly Active Users. This metric is used to evaluate the "health" of a service, because in a data driven market, the constant influx of information is an indicator of growth, and growth is what the market wants. Active users impact the services on three levels. Firstly, the more the active users, the larger the quantity of advertising that can be shown to them. Secondly, the more people rely on a service, the more information can be gathered from and about them and their habits, for various reasons such as better targeting, better service design, business intelligence, even service vacuum that may lead to new business opportunities (the infamous Data Mining and Big Data). And lastly, the MAU metric is a key indicator for investors, making it one of the drivers of share prices, and an especially important gauge for those companies that have gone public.

This is why services do their best to capture out attention - from notifications about messages from our friends (the most basic notification), to reminder notifications ("Just a reminder to step on the scale and update your current weight today" the calorie-tracking sports app "MyFitnessPal" tells me every once in a while), to marketing support messages ("People may be searching for your Page online. Write a post to keep your Page upto-date for new visitors", Facebook reminds us every so often).

This is where FOMO kicks in - the Fear Of Missing Out. Notifications are designed to take advantage of FOMO, since we are prone to react to such triggers - and once we do, we are, effectively, active users. We open the application and see an advert, we actively use the service and, in exchange, our devices send valuable information about ourselves and our behaviors back to its servers. Services have become much more easily accessible and user-friendly - after all, UX designers are hard at work. Using these apps and services has become fun, it's pleasurable - and not without intention. They are designed to be addictive.

In quite the same manner, we always desire the latest smartphone, lest we miss out on novel functionalities (not to mention technology as a status symbol). We feel an urge to upgrade to the latest version of the operating system, update and upgrade our applications... We ogle the newest wearable tech - so that we can acquire it as soon as possible - because who knows how much it could improve our lives! We always fear we are missing out on amazing potential. Naturally, design takes these propensities into account and exploits them as best it can.

\section{Attention \& Habit}

With the myriad services that seek our attention, it gets spread too thin - and is effectively shattered to pieces. We watch TV, look at our phones, 
chat with people, change the TV channel, read an article, pause reading to view a tweet, reply on messenger, open a forgotten tab to view a video, return to the article, check a term on Wikipedia and go on a deep wiki dive, following links from one article to the next... then we see a YouTube cat video - and all at the same time! This is infinite semiosis IRL (in real life)! We get used to distractions and lose our ability to concentrate, to fully immerse ourselves into individual tasks.

In fact, research suggests that skim reading - "the new normal" as a Guardian article ${ }^{5}$ puts it - has rendered us less empathetic and largely lacking the ability to analyze critically. We are losing the ability to activate "deep reading" processes, as Harvard University Prof. Maryanne Wolf refers to them - an "array of sophisticated processes that propel comprehension and that include inferential and deductive reasoning, analogical skills, critical analysis, reflection, and insight." (Wolf and Barzillai 2009) Our innate inclination to instant gratification is our Achilles' heel and contemporary design is taking full advantage of it.

UX design is usually perceived as "natural", since it is invisible (most people hardly give it any thought) and inevitable - after all, a service needs a way to interact with users. What UX design does (and that includes algorithms, AI, ML and deep learning - in their role of UX-serving tools) is that it indiscernibly and covertly introduces new habit-building paradigms of meaning-making into digital services. These paradigms have a profound effect on our culture - we could even venture as far as claiming that some of them turn into rituals (a hypothesis which we would further discuss with Prof. Reni Yankova, whose work on habit and ritual is remarkable ${ }^{6}$ ).

The ease of finding better options - actuating that desire for instant gratification, pushing us to realize the potential we may be missing - commodifies even our relationships (love, sex, affection). Jumping from one relationship to another, thanks to the affordances of dating apps, becomes increasingly easy. We could call this trait "fomosexuality" - the relationship equivalent of skim reading; moving on, based on FOMO. This development may be bringing to a society-sanctioned relaxation of social norms regarding sexuality and intimate relations - a societally-shared habit of laxness. A liberalization, an accidental, UX-design-driven sexual revolution. A re-writing of the sexual scripts (Gagnon and Simon 2005) as a side effect!

\footnotetext{
${ }^{5}$ https://www.theguardian.com/commentisfree/2018/aug/25/skim-reading-new-normalmaryanne-wolf

${ }^{6}$ See her book "Семиотични орбити“" [Semiotic Orbits], New Bulgarian University, 2018 .
} 
This turn of events has us wondering what could come next. Perhaps a modularization of relationships? From the intimate AiO to a collection of purpose-specific relationships? A purely sexual relationship; then a relationship for romance; another one for companionship, then one based on devotion; a relationship with somebody to love, and another one - to be loved by someone? Could this UX-driven liberalization influence gender equality? Would it lead to a broader recognition of gender fluidity? It is quite possible that we see such developments in the near future. What is certain is that UX design, even outside of the scope of its prescribed goals, outside its implicit and explicit objectives, has led to profound changes in our culture and will inevitably continue to do so; we can only strive to be mindful of these processes.

\section{References}

Anderson, Benedict. 2016. Imagined Communities: Reflections on the Origin and Spread of Nationalism. New York: Verso.

Bankov, Kristian. 2010. Cultures of navigation versus cultures of erudition. Lexia Vol. I (September 2010), 103-123.

Bankov, Kristian. 2017. "Eco and the Google Search Innovations" in Thellefsen, T. and Sørensen, B. (eds) Umberto Eco in His Own Words, Berlin: De Gruyter Mouton, pp. 119-126.

Blank, Grant \& Reisdorf, Bianca. 2012. The Participatory Web. Information. 15.

DiNucci, Darcy. 1999. "Fragmented Future” (PDF). Print. 53 (4): 32.

Eco, Umberto. 1978. A Theory of Semiotics. Bloomington: Indiana University Press.

Eco, Umberto. 1979. The Role of the Reader. Bloomington: Indiana University Press.

Eco, Umberto. 1986. Semiotics and the Philosophy of Language. Bloomington: Indiana University Press.

Gagnon, J. H. \& Simon, W. (2005). Sexual conduct: The social sources of human sexuality. New Brunswick, NJ: Aldine Transaction. 
Knight, Will. 2017. The Dark Secret at the Heart of AI. (last accessed January 2018 from https://www.technologyreview.com/s/604087/thedark-secret-at-the-heart-of-ai/).

Kosinski, Michal, Stillwell, David \& Graepel Thore. Digital records of behavior expose personal traits. Proceedings of the National Academy of Sciences Apr 2013, 110 (15) 5802-5805.

Maryanne Wolf, Mirit Barzillai. 2009. The Importance of Deep Reading. Educational Leadership Vol. 66 (March 2009), 32-37.

Milan, Stefania. 2015. When Algorithms Shape Collective Action: Social Media and the Dynamics of Cloud Protesting. Social Media + Society Vol II (2015).

Orszag, Peter. 2017. People Lie, But Search Data Tell the Truth. (last accessed January 2018 from https://www.bloomberg.com/view/articles/2017-05-09/people-lie-but-search-data-tell-the-truth).

Pariser, Eli. The Filter Bubble: What the Internet is Hiding from You. London: The Penguin Press, 2011. Ebook.

Rieder, Bernhard. Democratizing Search? From Critique to Society-oriented Design.. Deep Search. The Politics of Search beyond Google., StudienVerlag / Transaction Publishers, pp.133-151, 2009.

Rieder, Bernhard. 2005. Networked Control: Search Engines and the Symmetry of Confidence. International Review of Information Ethics Vol. III (June 2005), 26-32.

Sieckenius de Souza, Clarisse. 2004. The Semiotic Engineering of Human-Computer Interaction. Cambridge: The MIT Press.

Srnicek, Nick. 2017. Platform Capitalism. Cambridge: Polity Press.

Vuzharov, Mihail. Personalization Algorithms - Limiting the Scope of Discovery? How algorithms force out serendipity. Digital Age in Semiotics and Communication Vol. I (2018), 19-33. 